Check for updates

Cite this: Mater. Chem. Front. 2020, 4, 775

Received 1st December 2019, Accepted 27th December 2019

DOI: 10.1039/c9qm00727j

rsc.li/frontiers-materials

\section{GeSe thin-film solar cells}

\author{
Shun-Chang Liu, ${ }^{\mathrm{ab}}$ Yusi Yang, ${ }^{\mathrm{a}}$ Zongbao Li, ${ }^{\mathrm{Cd}}$ Ding-Jiang Xue (D) *ab and \\ Jin-Song Hu (D) *ab
}

\begin{abstract}
Thin-film solar cells made from non-toxic and earth-abundant materials are needed to substitute the current best-developed absorbers such as cadmium telluride ( $\mathrm{CdTe}$ ) and copper indium gallium selenide (CIGS) due to the toxicity of $\mathrm{Cd}$ and scarcity of In and Te. In this aspect, germanium monoselenide (GeSe) satisfies the aforementioned criteria and has recently emerged as a potential replacement. Moreover, GeSe possesses a suitable bandgap of $1.14 \mathrm{eV}$ (optimal for single junction solar cells), high absorption coefficient $\left(>10^{5} \mathrm{~cm}^{-1}\right)$ at a wavelength close to the absorption onset, high hole mobility $\left(128.6 \mathrm{~cm}^{2} \mathrm{~V}^{-1} \mathrm{~s}^{-1}\right)$, and simple binary composition with fixed orthorhombic phase at room temperature. This review introduces the properties of GeSe with special emphasis on the material, optical and electrical properties, and then summarizes the recent progress of GeSe-based solar cells. Finally, we give guidance on optimizing GeSe thin-film solar cells to their full performance potential, and provide a brief outlook for the further development of GeSe thin-film solar cells.
\end{abstract}

\section{Introduction}

Currently, crystalline-Si (c-Si) solar cells including polycrystalline and monocrystalline Si rule the photovoltaic (PV) market owing to their relatively high power conversion efficiencies (PCEs), excellent stability and reliability, as well as the

\footnotetext{
${ }^{a}$ Beijing National Laboratory for Molecular Sciences (BNLMS), CAS Key Laboratory of Molecular Nanostructure and Nanotechnology, Institute of Chemistry, Chinese Academy of Sciences, Beijing 100190, China. E-mail: djxue@iccas.ac.cn, hujs@iccas.ac.cn

${ }^{b}$ University of Chinese Academy of Sciences, Beijing 100049, China

${ }^{c}$ School of Material and Chemical Engineering, Tongren University, Tongren 554300, China

${ }^{d}$ National Engineering Research Center for Advanced Polymer Processing Technology, Zhengzhou University, Zhengzhou, 450002, China
}

well-established Si industry. ${ }^{1-4}$ The c-Si solar cells account for about $94 \%$ of the total annual production. ${ }^{5}$ However, due to the low absorption coefficient of $\mathrm{Si}$ originated from its indirect bandgap, c-Si can only be used for solar cells with a thickness of about $200 \mu \mathrm{m}$ to absorb most of the incident light. ${ }^{3,6-8}$ This limits their applications in flexible and building integrated devices; this high material consumption has also been considered to be the main factor limiting further cost reduction.

In terms of the use of absorber materials with high absorption coefficient, thin-film solar cells offer the possibility to fabricate flexible devices on flexible substrates such as metal and polyimide films while reducing the material consumption. ${ }^{8}$ At present, the most representative thin-film solar cells are cadmium telluride (CdTe) and copper indium gallium selenide (CIGS). Their PCEs have reached $22.1 \%{ }^{9}$ and $23.4 \%,{ }^{10}$ respectively.

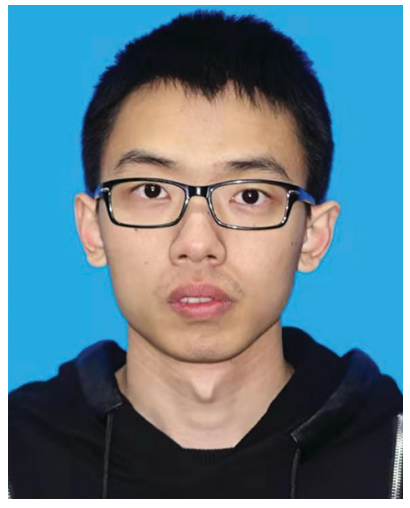

Shun-Chang Liu received his BS degree in Chemistry from Nankai University in 2015. He is now a PhD candidate at the Institute of Chemistry, Chinese Academy of Sciences (ICCAS) under the supervision of Prof. Jin-Song $\mathrm{Hu}$, and has been since 2015. His research focuses on GeSe thinfilm solar cells and inorganic perovskite solar cells.

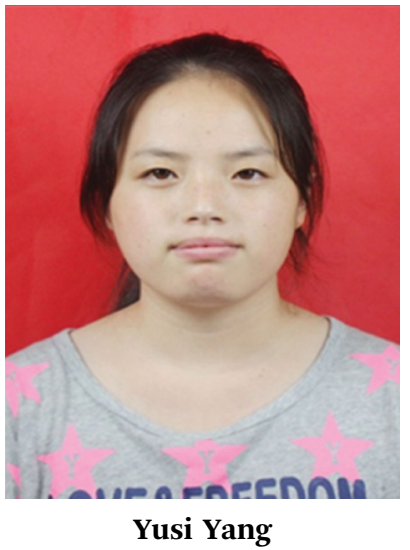

Yusi Yang is currently a PhD student candidate of Key Laboratory for Physics and Chemistry of Nanodevices in the Department of Electronics at Peking University. She also joined Prof. Jin-Song Hu's group at Institute of Chemistry, Chinese Academy of Sciences (ICCAS) to finish her $P h D$ research. Her current research interests focus on anisotropic two-dimensional materials. 
Notably, flexible CIGS solar cells grown on polyimide substrates exhibited a PCE of $18.7 \%$, indicating that flexible thin-film solar cells with high efficiencies comparable to those on rigid substrates can be achievable. ${ }^{11}$ However, the above bestdeveloped thin-film absorber materials have fundamental limitations when it comes to mass-scale production due to the toxicity of $\mathrm{Cd}$ in CdTe and the scarcity of In in CIGS. ${ }^{12}$ Moreover, halide perovskites, the hottest research topic in the $\mathrm{PV}$ field, suffer from the toxicity of $\mathrm{Pb}$ and severe stability problems, although they have achieved a PCE of $25.2 \%$ over the past decade. , $^{9-15}$

Therefore, besides the excellent optoelectronic properties, an ideal candidate for thin-film solar cells should satisfy the following criteria: (i) no harm to the environment; (ii) earth abundance; (iii) excellent stability. Many absorber materials satisfying these requirements have been explored, such as $\mathrm{SnS},{ }^{16-20} \mathrm{Sb}_{2}(\mathrm{~S}, \mathrm{Se})_{3},{ }^{21-27} \mathrm{CuSbSe}_{2}{ }^{28,29}$ and $\mathrm{Cu}_{2} \mathrm{O}^{30-32}$ Among such kind of materials, germanium monoselenide (GeSe) has received more and more attention recently. The first GeSe thin-film solar cell with an efficiency of $1.48 \%$ was reported in $2017 .{ }^{33}$ Considering the high theoretical Schockley-Quiesser efficiency limit of nearly $30 \%$ for GeSe single junction solar

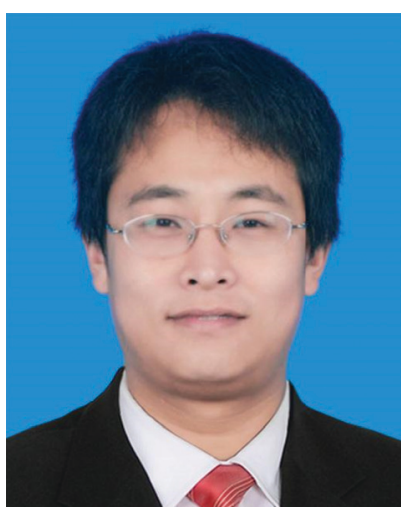

Zongbao Li
Zongbao $\mathrm{Li}$ received his $\mathrm{PhD}$ degree from South China Normal University in 2019. Currently, he is a Professor in Tongren University. His current research interest focuses on new two-dimensional functional materials.

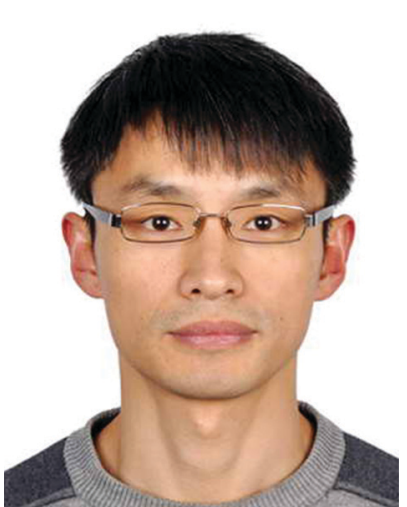

Ding-Jiang Xue
Ding-Jiang Xue received his $P h D$ degree from the Institute of Chemistry, Chinese Academy of Sciences (ICCAS) in 2013. Currently, he is a Professor in ICCAS. His research interest focuses on inorganic thin-film solar cells based on GeSe and inorganic perovskites. cells, there is still tremendous scope to further improve the PCE of GeSe thin-film solar cells. ${ }^{34}$ This review is going to comprehensively introduce the properties of GeSe, summarize the recent progress of GeSe thin-film solar cells, and identify the problems existing in the development of GeSe thin-film solar cells.

\section{Properties of GeSe}

GeSe is a member of group-VI monochalcogenides ( $\mathrm{SnS}$, SnSe, GeS and GeSe), known as phosphorene analogues. ${ }^{35-38}$ Recently, GeSe has been widely investigated in photodetectors, ${ }^{39-46}$ ovonic threshold switching devices, ${ }^{47-50}$ PEC water splitting, ${ }^{51}$ gas sensors, ${ }^{52}$ field effect transistors (FETs) ${ }^{53-55}$ and photovoltaics. ${ }^{3,56,57}$ Among these applications, GeSe displays great potential in the field of photovoltaics due to its excellent material, optical and electrical properties. In this section, we will review the fundamental properties of GeSe, including material, optical and electrical properties.

\subsection{Material properties}

GeSe has an orthorhombic crystal structure with Pnma 62 space group at room temperature. The corresponding lattice parameters are $a=4.40 \AA, b=3.83 \AA$, and $c=10.84 \AA$, respectively. ${ }^{58}$ This phase undergoes a phase transition to the cubic Fm $3 m$ structure $(a=5.76 \AA)$ at $651{ }^{\circ} \mathrm{C} .{ }^{59}$ The melt point of GeSe is $670{ }^{\circ} \mathrm{C}$, higher than that of $\mathrm{Sb}_{2} \mathrm{Se}_{3}\left(612{ }^{\circ} \mathrm{C}\right)$ while far lower than that of CdTe $\left(1093{ }^{\circ} \mathrm{C}\right) .{ }^{12,59-62}$ Considering the high phase transition temperature, our discussion mainly focusses on the thermodynamically preferred orthorhombic phase at room temperature.

As a layer material, GeSe consists of double-layer slabs separated from one another by weak van der Waals (vdW) forces along the $z$ axis (Fig. 1a). ${ }^{33,63}$ Particularly, there are two nonequivalent in-plane crystal directions of GeSe: armchair and zigzag. ${ }^{64}$ This is originated from the puckered atom structure, 3-fold covalently coordinated Ge-Se (Fig. 1b). To quantify the

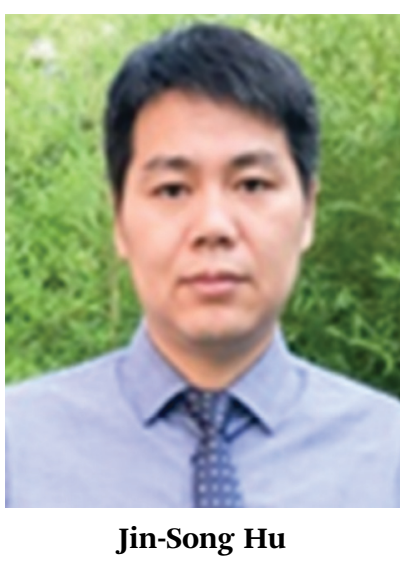

Jin-Song $\mathrm{Hu}$ is currently a professor at the Institute of Chemistry, Chinese Academy of Sciences (ICCAS). He received his $P h D$ degree in Physical Chemistry at ICCAS in 2005, then joined ICCAS as an assistant professor and was promoted as an associate professor in 2007. He worked in professor Charles $M$. Lieber's group at Harvard University in 2008-2011, then moved back to ICCAS as a Full Professor. His research currently focuses on developing new functional nanomaterials for efficient electrochemical energy conversion and solar energy conversion. 
(a)

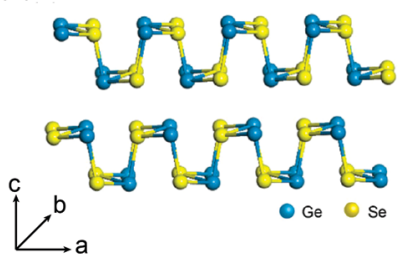

(b)

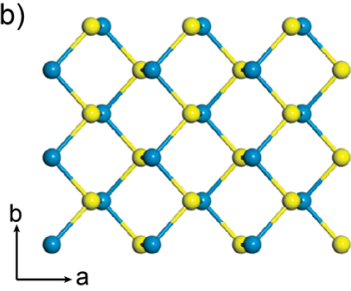

(c)

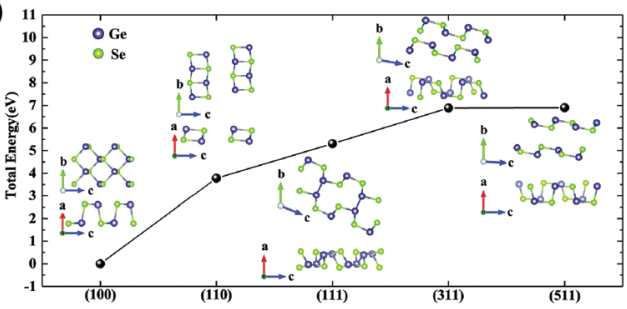

Fig. 1 Crystal structure of orthorhombic GeSe from (a) side view and (b) top view. Reprinted with permission from ref. 33. Copyright 2017, American Chemical Society. (c) The total energy of different crystal surfaces. The value of the plane (100) is set to zero as the reference. Reprinted with permission from ref. 56. Copyright 2019, Royal Society of Chemistry.

covalency of the Ge-Se bond, Bader charge analysis was performed by Xia et $a l .{ }^{65}$ The result showed that one Ge atom donated only $0.66 \mathrm{e}$ per Se atom, while one $\mathrm{Sn}$ atom donated 0.95 e per $\mathrm{S}$ atom in SnS. This indicates the very strong covalent character of GeSe. It is originated from the little difference in electronegativity between Se (2.55) and Ge (2.01). ${ }^{66}$

The layered crystal nature makes GeSe tend to form 2D nanosheets in the synthesis process. This can be attributed to different surface energies shown in Fig. 1c. ${ }^{57}$ This strong anisotropy of crystal structure inevitably leads to the anisotropic optical and electrical properties of GeSe. Therefore, engineering the crystal structure with preferential orientation plays a critical role in the device performance for photovoltaic application. ${ }^{22,67,68}$

In contrast to CdTe and lead perovskite, the raw material of GeSe is non-toxic. Although excessive exposure to Ge needs to be avoided, the toxicity of Ge is very low. ${ }^{58} \mathrm{GeSe}$ is absent from the list of highly toxic or carcinogenic materials by regulation authorities of China, America or the EU. The elemental abundances of Ge and Se in crustal rock are $1.5 \mathrm{ppm}$ and $0.05 \mathrm{ppm}$, respectively. They are both higher than the abundance of Te $(0.005 \mathrm{ppm})$ and In (0.049 ppm), making the massive production of GeSe solar cells possible. ${ }^{12}$

\subsection{Optical properties}

Optoelectronic properties are very important for photovoltaic applications. GeSe has emerged as a promising absorber material owing to its excellent optoelectronic properties. GeSe is an indirect-bandgap semiconductor with a bandgap of $1.14 \mathrm{eV}$ (Fig. 2a), while the difference between indirect bandgap and direct bandgap $(1.21 \mathrm{eV})$ is very small. ${ }^{58,69}$ This bandgap is optimal for single junction solar cells, enabling a ShockleyQueisser efficiency limit of $\sim 30 \% .{ }^{34}$ Combined with ultraviolet photoelectron spectroscopy (UPS), the valence band maximum (VBM) and conduction band minimum (CBM) are determined
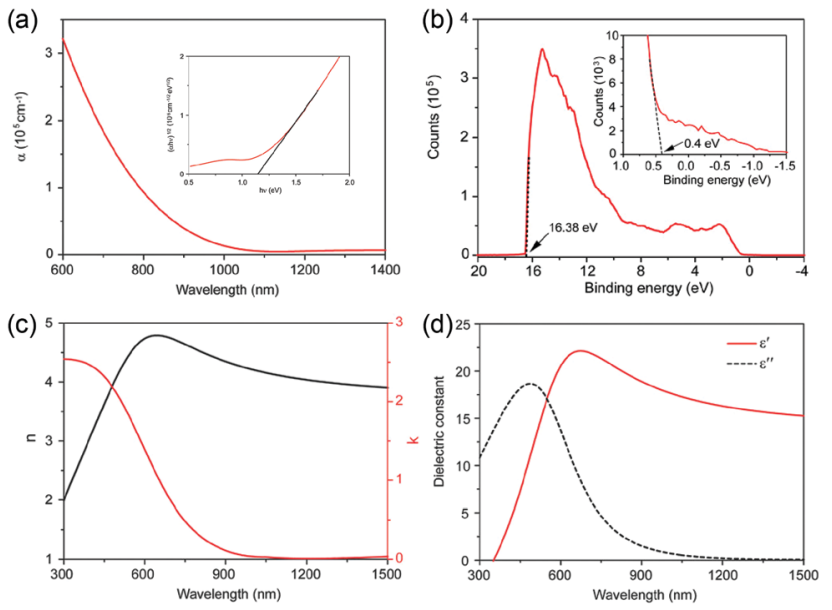

Fig. 2 (a) Absorption coefficient of GeSe film. Inset: Tauc plot for GeSe film $\left(E_{\mathrm{g}}=1.14 \mathrm{eV}\right)$. (b) UPS spectrum of GeSe film. Reprinted with permission from ref. 33. Copyright 2017, American Chemical Society. (c) Refractive index (black line) and extinction coefficient (red line) of GeSe in the wavelength range from 300 to $1500 \mathrm{~nm}$. (d) Real (red line) and imaginary (black dash line) parts of the relative dielectric constant of GeSe calculated from (c). Reprinted with permission from ref. 69. Copyright 2017, Wiley-VCH.

to be $-5.23 \mathrm{eV}$ and $-4.09 \mathrm{eV}$, respectively; the Fermi level is located at $-4.83 \mathrm{eV}^{33}$

GeSe shows a high absorption coefficient $\left(>10^{5} \mathrm{~cm}^{-1}\right.$ in the visible region) as shown in Fig. 2a. ${ }^{69}$ Specifically, visible light can be totally absorbed by GeSe film within a thickness of $460 \mathrm{~nm}$. The refractive index $(n)$ and extinction coefficient $(k)$ of GeSe were tested using an ellipsometer by our group. The measured $n$ and $k$ are displayed in Fig. 2c. The reflectivity and relative dielectric constant are calculated from $n$ and $k$. The calculated reflectivity is about $40 \%$. This relatively high reflection loss indicates that antireflective coatings are necessary for GeSe solar cells. The dielectric constant is another important parameter for semiconductors (following formula: $\varepsilon^{\prime}=n^{2}-k^{2}$, $\varepsilon^{\prime \prime}=2 n k$, where $\varepsilon^{\prime}$ and $\varepsilon^{\prime \prime}$ are real and imaginary parts of relative dielectric constant, respectively). ${ }^{70}$ The dielectric constant is related to exciton binding energy. The calculated relative dielectric constant of GeSe is 15.3. This is even larger than CIGS (15.2) and CdTe (10.0), ${ }^{71}$ indicating the low exciton binding energy in GeSe, consistent with the calculated exciton binding energy $(0.21 \mathrm{eV}) .^{72}$ This low value endows the highly-efficient separation of electrons and holes, making GeSe suitable for the simple planar heterojunction thin-film solar cell architecture.

\subsection{Electrical properties}

The electrical properties of GeSe single crystals grown using different methods have been reported in 2003 by Solanki et al. as shown in Table $1 .^{73}$ GeSe normally exhibits p-type conductivity due to the native Ge vacancy. ${ }^{74}$ The best measured majority mobility is $128.7 \mathrm{~cm}^{2} \mathrm{~V}^{-1} \mathrm{~s}^{-1}$, higher than that of $\mathrm{CH}_{3} \mathrm{NH}_{3} \mathrm{PbI}_{3}$ single crystals. Kim et al. investigated the electrical properties of GeSe single crystals fabricated by using the Bridgman technique, obtaining a mobility of $71.5 \mathrm{~cm}^{2} \mathrm{~V}^{-1} \mathrm{~s}^{-1} \cdot{ }^{75}$ 
Table 1 Electrical properties of GeSe single crystals grown using different methods

\begin{tabular}{|c|c|c|c|c|c|}
\hline Crystal & Hall coefficient $R_{\mathrm{H}}\left(\mathrm{cm}^{3} \mathrm{C}^{-1}\right)$ & Carrier concentration $p\left(\mathrm{~cm}^{-3}\right)$ & Resistivity $\rho(\Omega \mathrm{cm})$ & Mobility $\mu\left(\mathrm{cm}^{2} \mathrm{~V}^{-1} \mathrm{~s}^{-1}\right)$ & Type \\
\hline $\mathrm{GeSe}\left(\mathrm{NH}_{4} \mathrm{Cl}\right)$ & 1330.45 & $4.7 \times 10^{15}$ & 13.36 & 99.57 & $\mathrm{p}$ \\
\hline $\operatorname{GeSe}\left(\mathrm{I}_{2}\right)$ & 24.36 & $2.43 \times 10^{17}$ & 2.0 & 12.26 & $\mathrm{p}$ \\
\hline
\end{tabular}

Our group also systematically investigated the electrical properties of polycrystalline GeSe thin film prepared by our rapid thermal sublimation method (this method will be discussed in detail in Section 3). ${ }^{69}$ The resistivity, majority mobility and concentration tested using the Van Der Pauw method are estimated to be $2.18 \times 10^{2} \Omega \mathrm{cm}, 14.85 \mathrm{~cm}^{2} \mathrm{~V}^{-1} \mathrm{~s}^{-1}$, and $1.93 \times 10^{15} \mathrm{~cm}^{-3}$, respectively. ${ }^{33}$ Compared with the reported highest majority mobility of GeSe single crystal, the low mobility of GeSe polycrystalline film could be due to the scattering of extra grain boundaries in the polycrystalline films. The space charge limited current (SCLC) method was further used to measure the electron and hole mobility of GeSe thin films (Fig. 3a and b). The hole and electron mobilities are estimated to be 10.4 and $11.2 \mathrm{~cm}^{2} \mathrm{~V}^{-1} \mathrm{~s}^{-1}$, respectively, in agreement with Hall effect measurements.

Carrier lifetime is another important parameter for photovoltaic absorber materials. Our group utilized transient absorption (TA) spectroscopy to measure the carrier lifetime of GeSe polycrystalline films. ${ }^{69}$ As shown in Fig. 3c, the wavelength of pulsed excitation was $400 \mathrm{~nm}$ and a different delay time was used for TA spectral evolution. The photoinduced absorption (PA) dynamic was fitted using a Gaussian response function and the carrier lifetime was estimated to be about $9.9 \mathrm{~ns}$ (Fig. 3d), close to that of $\mathrm{CH}_{3} \mathrm{NH}_{3} \mathrm{PbI}_{3}$ thin films (9.6 ns). ${ }^{76}$ Based on the measured carrier lifetime (9.9 ns) and minority mobility of GeSe film, the minority carrier (electron) diffusion length is obtained to be about $531 \mathrm{~nm}$. Therefore, the above
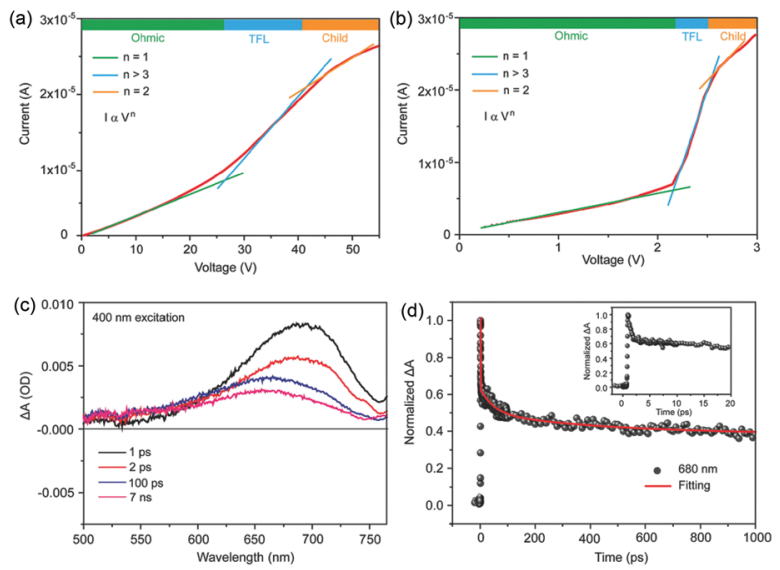

Fig. 3 Dark current-voltage curves for (a) a hole-only and (b) an electron-only GeSe device. (c) TA spectra for GeSe film with a pulsed excitation wavelength of $400 \mathrm{~nm}$ at different delays. (d) TA dynamics with a probe wavelength of $680 \mathrm{~nm}$ and the fitting curve using Gaussian response function convoluted with the triexponential decay function. Inset of (d) is the zoom in for the first 20 ps. Reprinted with permission from ref. 69. Copyright 2017, Wiley-VCH.

measured electrical properties merit the exploration of GeSe for high-performance thin-film solar cells.

\section{GeSe thin film fabrication}

Due to the great potential in photovoltaics, GeSe thin films had been prepared by vacuum thermal evaporation onto a stainlesssteel substrate as early as 1980, while the as-prepared GeSe film was amorphous. ${ }^{77}$ Since then, many literature studies about amorphous GeSe thin films have been reported. Kamboj et al. deposited GeSe thin films onto glass substrates at room temperature by the electron beam evaporation technique in $2002 .^{78}$ Bosse et al. fabricated GeSe phase change memory films by thermal co-evaporation of the Ge and Se source on the Si substrate in 2014. ${ }^{79}$ Recently, Kim et al. reported the deposition of GeSe films through the atomic layer deposition technique (Fig. $4 \mathrm{a}-\mathrm{c}$ ). ${ }^{80}$ There were no lattice fringes (Fig. $4 \mathrm{a}$ and b) or diffraction peaks (Fig. 4c) observed of the as-prepared film, demonstrating its disordered structure. However, amorphous GeSe is not suitable for photovoltaic applications due to the high defect density, low absorption coefficient and large bandgap.

To obtain polycrystalline GeSe thin films, there are usually two preparation methods. The first method is through the thermal annealing of amorphous GeSe film. The crystallization temperature of GeSe has been measured to be about $330{ }^{\circ} \mathrm{C}$ through X-ray diffraction (XRD) and differential thermal analysis (DTA) (Fig. 4d and e). ${ }^{81}$ There have been several reports about
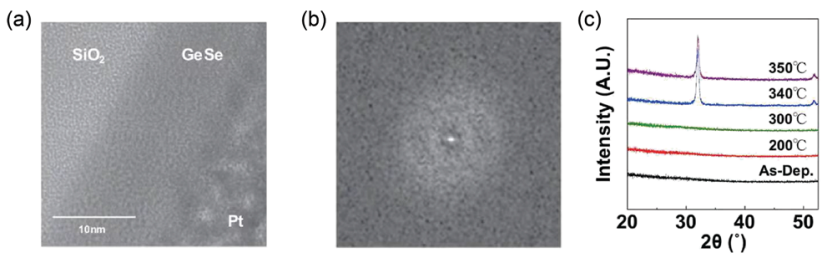

(d)
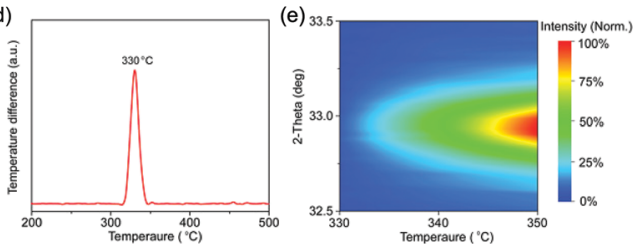

Fig. 4 (a) TEM image and (b) associated FFT analysis of GeSe grown through the ALD process. (c) XRD patterns of as-deposited and annealed GeSe grown through the ALD process. Reprinted with permission from ref. 80. Copyright 2018, IOP Publishing Ltd. (d) DTA of GeSe powder scraped from the as-prepared amorphous GeSe film grown by thermal evaporation. (e) Contour colour map of temperature-dependent XRD patterns of GeSe film grown by thermal evaporation. Reprinted with permission from ref. 81. Copyright 2018, Wiley-VCH. 
(a)
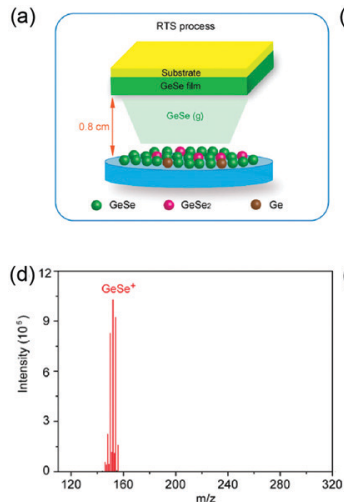

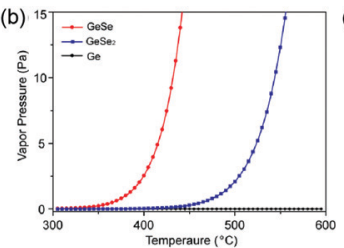

(e)

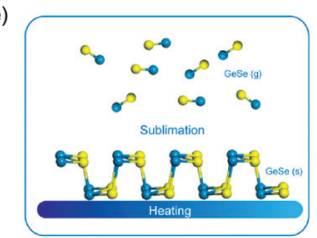

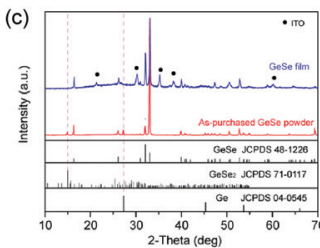

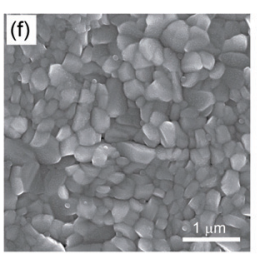

Fig. 5 (a) XRD patterns of as-purchased GeSe powder and GeSe thin film deposited by RTS on an ITO substrate. (b) Saturated vapor pressure of GeSe, $\mathrm{GeSe}_{2}$, and Ge from 300 to $600^{\circ} \mathrm{C}$. (c) Schematic diagram of RTS for depositing GeSe thin film. (d) The dominant components of vapor species sublimed from GeSe(s) at $400{ }^{\circ} \mathrm{C}$. (e) Simplified schematic of the sublimation mechanism of GeSe. (f) Top-view SEM image of GeSe thin film deposited by RTS. Reprinted with permission from ref. 33. Copyright 2017, American Chemical Society.

the fabrication of polycrystalline GeSe thin films through this method. However, these samples had always a residue of amorphous phase GeSe, and suffered from adverse film orientation, which may significantly influence the performance of GeSe solar cells.

Another route is the one-step fabrication from the as-prepared GeSe powder to crystalline film directly. Based on the sublimation feature of GeSe, our group first reported the fabrication of polycrystalline GeSe thin film through rapid thermal sublimation (RTS) (Fig. 5a). ${ }^{33}$ The unique advantage of this RTS method is that we achieve the deposition of crystalline film and purification of raw material of GeSe in one step. This is based on the different vapor pressures of GeSe and other impurities (Fig. 5b), enabling the desired GeSe to deposit onto the substrate while leaving the impurities in the source (Fig. 5c). This lowers the requirement of raw materials and hence reduces the cost of manufacturing. We further investigated the sublimation mechanism of GeSe through mass spectrometry. As shown in Fig. $5 d$, the main products in the vapor phase are the diatomic molecules $\operatorname{GeSe}(\mathrm{g})$. This stoichiometric sublimation style (Fig. 5e) can effectively suppress the formation of harmful point defects such as deep-level interstitials and antisites. The as-prepared GeSe thin films obtained by RTS show high quality with crystalized grains and free of cracks and pinholes as shown in the top-view scanning electron microscopy (SEM) image (Fig. 5f). Therefore, the above method for the fabrication of GeSe thin films lays the foundation for their photovoltaic application.

It is well-known that solution-processed photovoltaic technologies are a promising method to reduce the manufacturing cost, while providing a facile way to achieve doping or alloying. Unfortunately, all the reported preparation methods for GeSe thin films are based on the vacuum technique. Until now, there has been no report about the solution method for the fabrication of GeSe films. This may be due to the multivalent component and strong covalency of GeSe. The multivalence of Ge makes it hard to find stable Ge precursor sources; the strong covalent bonding makes it difficult to obtain the GeSe precursor solution. However, considering that many nanostructures of GeSe have been synthesized through the solution method, we think that solution-processed GeSe thin films will be achievable in the near future, thus providing a broad space for the in-depth investigation of GeSe. ${ }^{82-86}$

\section{GeSe-based solar cells}

\subsection{Theory simulation for GeSe photovoltaics}

The electronic configuration of GeSe has been extensively studied. The calculated bandgap of bulk GeSe is in the range of 1.04-1.20 eV (Fig. 6a), close to the experimental value of $1.14 \mathrm{eV} .^{33,35,40,87}$ The higher part of the valence band is composed of Ge-4s, Ge-4p and Se-4p states while the latter is dominating (Fig. 6b). ${ }^{88,89}$ The lower part of the conduction band is mainly made of hybridized states formed between Ge-4p and Se-4p states; the components of Ge-4p states are larger than that of Se-4p states. As a consequence of the unique electronic configuration, the joint density of states is large in GeSe and results in enhanced probability of optical transition, beneficial to light absorption. Therefore, the combination of large absorption coefficient and suitable bandgap makes GeSe promising as a light absorber material. (a)

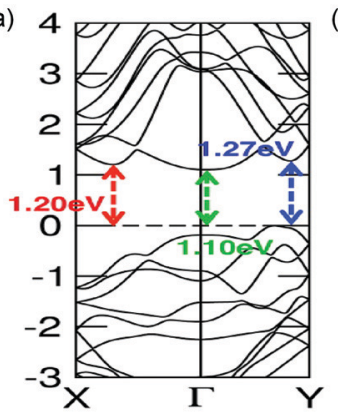

(b)

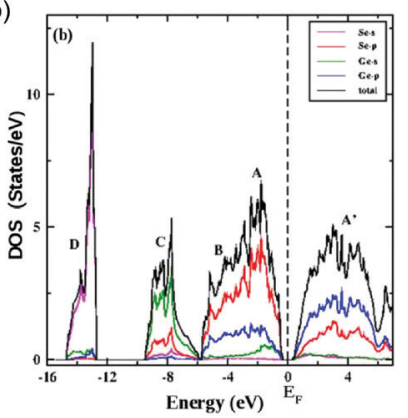

Fig. 6 (a) Band structure of bulk GeSe. Reprinted with permission from ref. 88. Copyright 2015, American Chemical Society. (b) Projected density of states for bulk GeSe. Reprinted with permission from ref. 89. Copyright 2015, IOP Publishing Ltd. 
In addition to the calculation about the band structure, there have been many reported calculations for GeSe thin-film solar cells directly. Shi et al. calculated the theoretical limit of GeSe for ultrathin solar-cell applications. The estimated upper limit to PCE to lowest exciton energy for single-layer GeSe and doublelayer GeSe is $5.2 \%$ and $7.2 \%$, respectively. ${ }^{88}$ It is comparable to the current efficiency of many other inorganic thin-film solar cells, such as $\mathrm{SnS}$ and $\mathrm{Sb}_{2} \mathrm{Se}_{3}$, indicating that GeSe is a promising material for efficient thin-film solar cells.

Furthermore, GeSe can form a heterojunction with other group-IV monochalcogenides to improve their photovoltaic performance. This is due to their special optoelectronic properties and good lattice match. Taking SnS for example, Xia et al. constructed a 2D vdW heterostructure by stacking GeSe and SnS monolayers, and then calculated the electronic properties of this GeSe/SnS heterobilayer. ${ }^{65}$ The GeSe/SnS heterobilayer exhibits a direct bandgap of $1.519 \mathrm{eV}$ (Fig. 7a). The CBM is mainly composed of GeSe, while the VBM is formed by about 91\% SnS and 9\% GeSe. From the band alignment of the GeSe/ SnS heterobilayer (Fig. 7b), it is obvious that GeSe and SnS monolayers form a type-II vdW heterostructure, where the band offsets, $\Delta E_{\mathrm{c}}$ and $\Delta E_{\mathrm{v}}$, of the heterobilayer are $0.147 \mathrm{eV}$ and $0.289 \mathrm{eV}$, respectively. These indicate that it can effectively separate holes and electrons. The Bader charge analysis also demonstrates that there are 0.016 electrons transferring from the SnS layer to the GeSe layer. According to this result, Lv et al. proposed a photovoltaic system based on GeSe/SnS (Fig. 7c). ${ }^{90}$ The PCE of the GeSe/SnS heterostructure was evaluated based on the method reported by Scharber et al..$^{91}$ This photovoltaic system shows a PCE of $\sim 18 \%$ (Fig. $7 d$ ), comparable to the certified efficiency of inorganic perovskite solar cells. Similarly, the
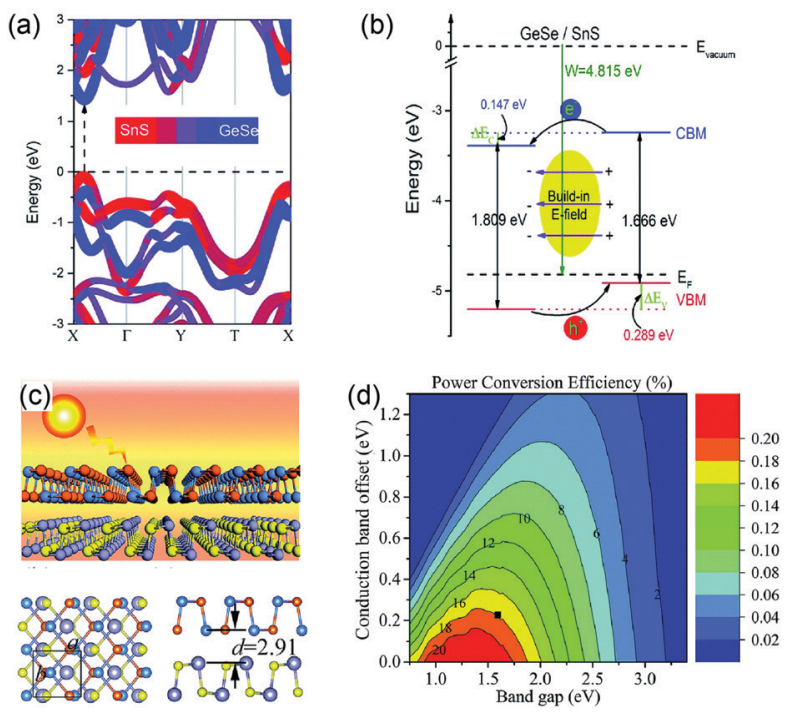

Fig. 7 (a) The projected band structures of the GeSe/SnS heterobilayer. (b) The band alignment of the GeSe/SnS heterobilayer. Reprinted with permission from ref. 64. Copyright 2017, Royal Society of Chemistry. (c) Schematic diagram of the GeSe/SnS heterobilayer. (d) Computed PCE contour as a function of the donor's band gap and CBO. Reprinted with permission from ref. 90. Copyright 2018, Royal Society of Chemistry.
GeSe/SnSe heterostructure was also studied by Mao et al. ${ }^{92}$ The GeSe/SnSe heterostructure exhibits excellent optoelectronic properties and good band alignment. Thanks to a smaller conduction band offset $(0.06 \mathrm{eV})$, the PCE of this system reaches a higher value $(21.47 \%)$ than that of the GeSe/SnS heterobilayer. The above predicted PCEs of the two heterostructure systems demonstrate that GeSe has enormous potential in the photovoltaic field, especially in flexible solar cells due to its proper band structure and excellent optoelectronic properties.

GeSe with a bandgap of $1.14 \mathrm{eV}$ is also a good choice to broaden the absorption spectrum of perovskite solar cells. The absorption edge of lead halide perovskite, i.e. $\mathrm{CH}_{3} \mathrm{NH}_{3} \mathrm{PbI}_{3}$, is about $800 \mathrm{~nm}$, resulting in the non-utility of near-infrared radiation. Hou et al. introduced a GeSe thin film into the perovskite $\left(\mathrm{CH}_{3} \mathrm{NH}_{3} \mathrm{PbI}_{3}\right)$ solar cell with a bilayer $\mathrm{p}-\mathrm{n}$ heterojunction structure of glass/ITO/PCBM/ $\mathrm{CH}_{3} \mathrm{NH}_{3} \mathrm{PbI}_{3}(350 \mathrm{~nm}) / \mathrm{GeSe}(450 \mathrm{~nm}) /$ PCDTBT/Ag (Fig. 8a) for theoretical simulation. ${ }^{93}$ According to the calculated absorption curve, the light absorptions of $\mathrm{CH}_{3} \mathrm{NH}_{3} \mathrm{PbI}_{3}$ and GeSe were exactly complementary to each other, successfully broadening the absorption edge to about $1200 \mathrm{~nm}$ (Fig. 8b). This was further confirmed by the quantum efficiency data (Fig. 8c). Fig. 8d showed the equilibrium band profile of the $\mathrm{CH}_{3} \mathrm{NH}_{3} \mathrm{PbI}_{3} /$ GeSe heterojunction. It was clear that there was a small barrier of $0.1 \mathrm{eV}$ between $\mathrm{CH}_{3} \mathrm{NH}_{3} \mathrm{PbI}_{3}$ and GeSe due to the offset of the conduction band edges. Considering that the barrier was lower than $0.4 \mathrm{eV}$, the negative influence on carrier collection was negligible. Therefore, the bilayer heterojunction could lead to effective dissociation of electrons and holes. The PCE of the $\mathrm{CH}_{3} \mathrm{NH}_{3} \mathrm{PbI}_{3} / \mathrm{GeSe}$ bilayer solar cell was calculated to be $23.77 \%$, much higher than that of $\mathrm{CH}_{3} \mathrm{NH}_{3} \mathrm{PbI}_{3}$ solar cells (16.66\%) (Fig. 8e). The relevant parameters are listed in Table 2. Specifically, the short circuit current density was improved significantly from $18.53 \mathrm{~mA} \mathrm{~cm}^{-2}$ to $37.62 \mathrm{~mA} \mathrm{~cm}^{-2}$, which was attributed to the wider light absorption range.

The above calculated results display the excellent optoelectronic properties of GeSe, and demonstrate the enormous potential of GeSe for thin-film photovoltaic applications. In the next subsection, we will summarize the recent progress in GeSe thin-film solar cells from experiments.

\subsection{GeSe thin-film solar cells}

The first GeSe solar cell was reported by our group in $2017 .^{33}$ We built a GeSe thin-film solar cell with a superstrate structure (ITO/CdS/GeSe/Au), as shown in Fig. 9a. The GeSe layer was prepared using the RTS method. The self-purified process and stoichiometric sublimation style of the RTS method ensure the high-quality of as-prepared GeSe films. The best-performing device exhibited an efficiency of $1.48 \%$, with an open circuit voltage $\left(V_{\mathrm{OC}}\right)$ of $0.24 \mathrm{~V}$, short-circuit current density $\left(J_{\mathrm{SC}}\right)$ of $14.48 \mathrm{~mA} \mathrm{~cm}^{-2}$, and fill factor (FF) of $42.60 \%$ (Fig. 9b). Considering the high absorption coefficient and ideal bandgap of GeSe, the loss of $J_{\mathrm{SC}}$ was a bit large, which was further verified by the low value of EQE (Fig. 9c). This may be attributed to the high concentration of interfacial defects between GeSe and CdS, as evidenced by the low rectification from the dark $J-V$ curve. Moreover, as a 2D layered material, the unfavourable 

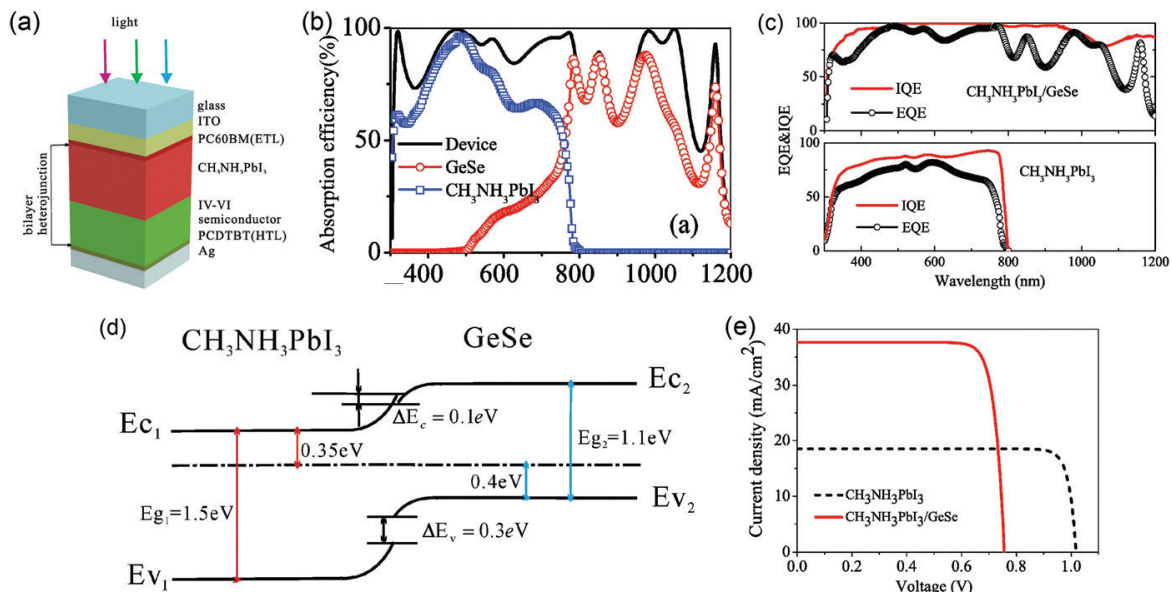

Fig. 8 (a) Schematic structure of the $\mathrm{CH}_{3} \mathrm{NH}_{3} \mathrm{Pbl}_{3} / \mathrm{GeSe}$ bilayer solar cell. (b) The calculated absorption spectrum for the device of the bilayer solar cell. (c) External quantum efficiency and internal quantum efficiency of the $\mathrm{CH}_{3} \mathrm{NH}_{3} \mathrm{Pbl}_{3} / \mathrm{GeSe}$ bilayer solar cell and $\mathrm{CH}_{3} \mathrm{NH}_{3} \mathrm{Pbl}_{3}$ solar cell. (d) The band alignment of the $\mathrm{CH}_{3} \mathrm{NH}_{3} \mathrm{Pbl}_{3} / \mathrm{GeSe}$ bilayer. (e) Current density-voltage $\left(\mathrm{J}-\mathrm{V}\right.$ ) curves of the $\mathrm{CH}_{3} \mathrm{NH}_{3} \mathrm{Pbl}_{3} / \mathrm{GeSe}$ bilayer solar cell and $\mathrm{CH}_{3} \mathrm{NH}_{3} \mathrm{Pbl}$ solar cell. Reprinted with permission from ref. 93. Copyright 2017, Elsevier.

Table 2 The relevant electrical quantities of a perovskite/GeSe bilayer solar cell and conventional perovskite solar cell

\begin{tabular}{lllll}
\hline Device type & $J_{\mathrm{SC}}\left(\mathrm{mA} \mathrm{cm}^{-2}\right)$ & $V_{\mathrm{OC}}(\mathrm{V})$ & $\mathrm{FF}(\%)$ & Efficiency (\%) \\
\hline Perovskite/GeSe & 37.62 & 0.76 & 83.14 & 23.77 \\
Perovskite & 18.53 & 1.02 & 88.15 & 16.66
\end{tabular}

(a)
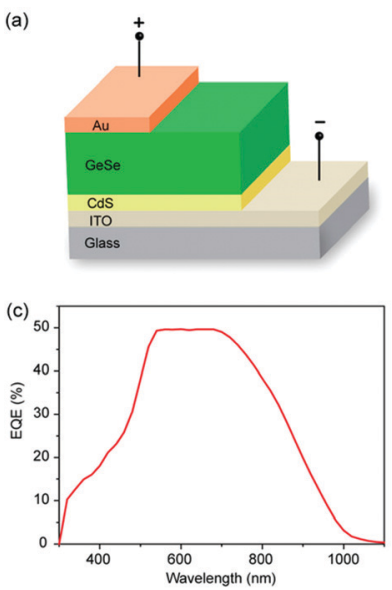
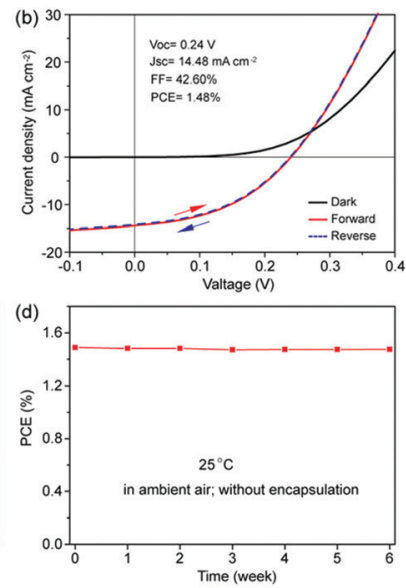

Fig. 9 (a) Schematic configuration of the GeSe solar cell. (b) Forward and reversed $J-V$ curves of the GeSe solar cell in the dark and under $100 \mathrm{~mW} \mathrm{~cm}^{-2}$ irradiation, respectively. (c) EQE spectrum of the GeSe solar cell. (d) Stability of a typical device without encapsulation stored under regular laboratory conditions. Reprinted with permission from ref. 33. Copyright 2017, American Chemical Society.

orientation of the GeSe thin film resulted in the insufficient photogenerated carrier collection. As for $V_{\mathrm{OC}}$, the $V_{\mathrm{OC}}$ deficit $\left(E_{\mathrm{g}} / q-V_{\mathrm{OC}}\right)$ was quite large, reaching up to $0.9 \mathrm{~V}$. The main reason for this large $V_{\mathrm{OC}}$ deficit may be the numerous interfacial defects. They can lead to the pinning of the Fermi level or unbefitting band alignment between GeSe and CdS. However, compared with perovskite solar cells, GeSe solar cells have two distinct advantages: (i) there was no hysteresis effect in GeSe solar cells (Fig. 9b), which was attributed to strong covalent character; (ii) GeSe solar cells showed excellent stability under a laboratory environment, with no obvious degradation after 6 weeks without any encapsulation (Fig. 9d).

Moreover, Chen et al. reported the GeSe thin-film solar cells fabricated by post annealing of amorphous GeSe films. ${ }^{56}$ The amorphous films were first deposited through magnetron sputtering (Fig. 10a). Then, they obtained the pure-phase and uniform GeSe thin films through regulating the annealing temperature; the optimum temperature was at $400{ }^{\circ} \mathrm{C}$ (Fig. 10b). Finally, they adopted carbon as a hole transport layer with a superstrate structure of SLG/FTO/CdS/GeSe/carbon/Ag, attempting to improve the carrier collection efficiency. However, the $J_{\mathrm{SC}}$ of this (a)
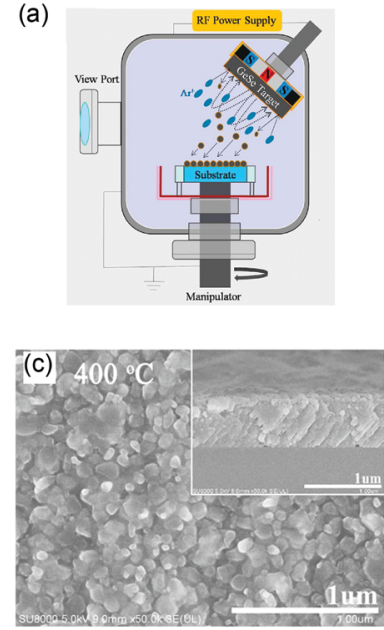

(b)

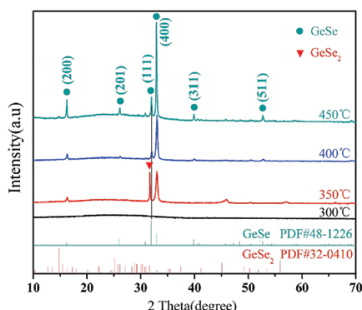

(d)

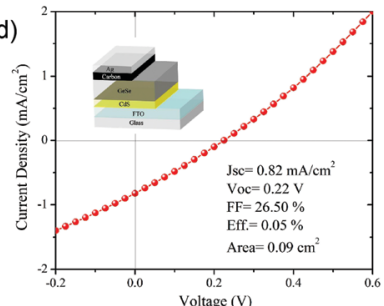

Fig. 10 (a) Schematic diagram of a magnetron sputtering system for GeSe preparation. (b) XRD patterns of GeSe thin films after annealing at different temperatures. (c) SEM images of GeSe thin film after annealing at $400{ }^{\circ} \mathrm{C}$. (d) J-V curves and relevant parameters of the GeSe solar cell (inset: the configuration of GeSe solar cell). Reprinted with permission from ref. 55. Copyright 2018, Elsevier. 
device was not as expected, as low as $0.82 \mathrm{~mA} \mathrm{~cm}^{-2}$. Therefore, the PCE of this device was only $0.05 \%\left(J_{\mathrm{SC}}=0.82 \mathrm{~mA} \mathrm{~cm} \mathrm{~cm}^{-2}\right.$, $V_{\mathrm{OC}}=0.22 \mathrm{~V}$ and $\mathrm{FF}=26.50 \%$ ) (Fig. 10d). The reason for this poor performance may be the low film quality. The GeSe thin films, fabricated by post annealing of amorphous GeSe, suffered from residue of amorphous phase and unfavourable orientation, as discussed in Section 3.

In addition, Zhang and Chen et al. proposed a new strategy to optimize the post-annealing procedure. ${ }^{57}$ A soda lime glass was placed on the as-synthesized amorphous GeSe film, which was prepared by thermal evaporation. Then, the glass and GeSe film were clamped together by a clamp and finally annealed in a tube furnace. The schematic diagram is shown in Fig. 11a. They claimed that this method, called sandwiching post-annealing treatment, was beneficial to suppress the sublimation of GeSe during the annealing process (Fig. 11b), thus improving the recrystallization process. Expectedly, the $J_{\mathrm{SC}}$ did improve greatly compared with their previous work, but it was still far away from the highest $J_{\mathrm{SC}}$ of GeSe solar cells reported so far. This method also inherently led to the unfavourable orientation (Fig. 11c) and low crystalline quality. The peak energy position of photoluminescence (PL), lower than the optical band gap, also demonstrated the existence of plenty of defects (Fig. 11d). In terms of device structure, the CdS buffer layer was replaced by $\mathrm{TiO}_{2}$, and the device adopted the superstrate configuration of $\mathrm{FTO} / \mathrm{TiO}_{2} /$ GeSe/carbon/Ag (inset of Fig. 11e). Compared with $\mathrm{CdS}, \mathrm{TiO}_{2}$ has three advantages: (i) $\mathrm{TiO}_{2}$ is nontoxic and more abundant; (ii) $\mathrm{TiO}_{2}$ has a wider bandgap of $3.6 \mathrm{eV}$, reducing the parasitic absorption; (iii) $\mathrm{TiO}_{2}$ exhibits excellent chemical stability. Fig. 11e shows the $J-V$ characteristics of the best GeSe solar cell based on a $\mathrm{TiO}_{2}$ buffer layer. The best PCE was $0.27 \%$ with a $J_{\mathrm{SC}}$ of $3.19 \mathrm{~mA} \mathrm{~cm}^{-2}$, a $V_{\mathrm{OC}}$ of $340 \mathrm{mV}$ and an $\mathrm{FF}$ of $25.15 \%$. Impressively, the $V_{\mathrm{OC}}$ of the champion device had a $42 \%$ increase compared with GeSe solar cells based on CdS counterparts. Encouragingly, the statistic $V_{\mathrm{OC}}$ showed that the best-performance $V_{\text {OC }}$ was even higher than $450 \mathrm{mV}$ (Fig. 11f), a surprising value for an emerging narrow-gap material. This may indicate a better band alignment or less interfacial defects between $\mathrm{TiO}_{2} / \mathrm{GeSe}$, compared with that of $\mathrm{CdS} / \mathrm{GeSe}$.

To evaluate the quality of the $\mathrm{TiO}_{2} / \mathrm{GeSe}$ heterojunction, capacitance-voltage $(C-V)$ and drive-level capacitance profiling (DLCP) technology were carried out. ${ }^{57}$ As shown in Fig. 12a, the doping density $\left(N_{\mathrm{A}}\right)$ of GeSe film built-in voltage $\left(V_{\mathrm{bi}}\right)$ can be derived using the following equation: ${ }^{94}$

$$
\frac{1}{C^{2}}=\frac{2\left(V_{\mathrm{bi}}-V\right)}{q A \varepsilon_{0} \varepsilon_{\mathrm{r}} N_{\mathrm{A}}}
$$

where $C, q, A, \varepsilon_{0}, \varepsilon_{\mathrm{r}}$, and $V$ represent capacitance, electron charge, electrode area, vacuum permittivity, relative permittivity, and bias voltage, respectively. The $N_{\mathrm{A}}$ (holes) of GeSe film was calculated to be $1.77 \times 10^{17} \mathrm{~cm}^{-3}$. The $V_{\mathrm{bi}}$ of the device was as high as $645 \mathrm{mV}$, larger than the value of $V_{\mathrm{OC}}$. The high $V_{\mathrm{bi}}$ may be benefitted from the good band alignment while the large loss of voltage might be due to the interfacial defects. $C-V$ profiling and DLCP measurements (Fig. 12b) were used to characterize the interfacial defects at the $\mathrm{TiO}_{2} / \mathrm{GeSe}$ interface. The depletion width of the GeSe device at zero bias was obtained as $138 \mathrm{~nm}$, far less than the thickness of the GeSe layer. This thin depletion width limited the collection of photogenerated carriers. Then, the interfacial defects were evaluated by the difference between $N_{C-V}$ and $N_{\text {DLCP. }}$. Since surface was relative to volume and width, the interfacial defect density was estimated to be $9.81 \times 10^{11} \mathrm{~cm}^{-2}$.
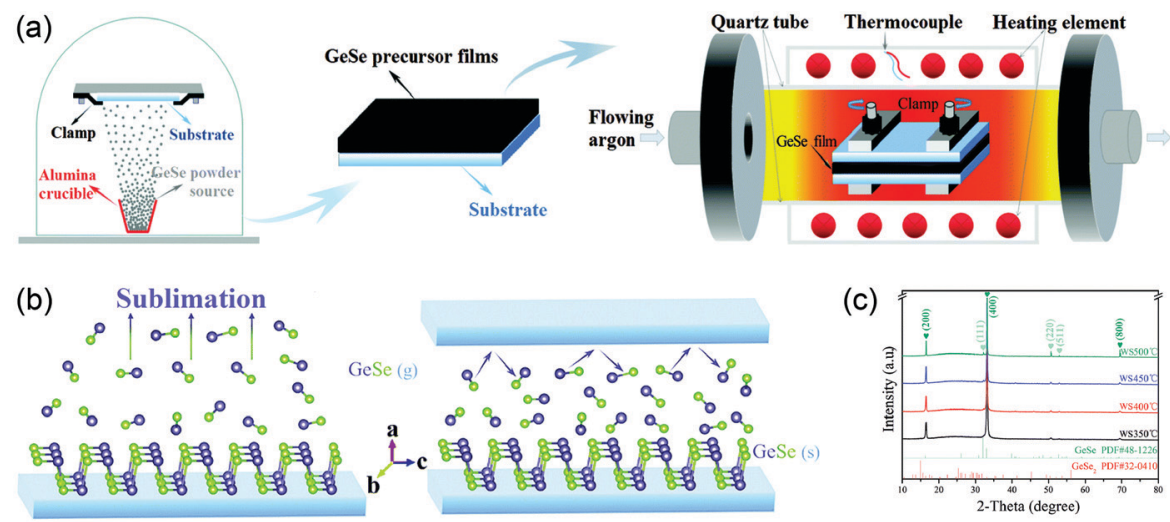

(c)

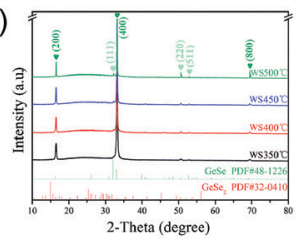

(d)

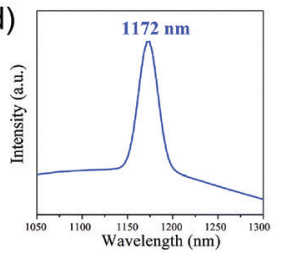

(e)

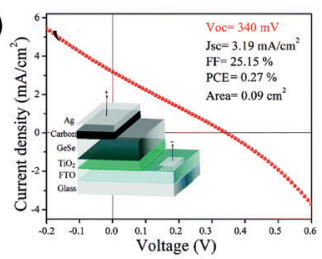

(f)

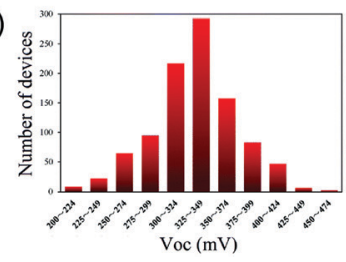

Fig. 11 (a) Schematic setup of the thermal evaporator and the sandwiching post-annealing process. (b) The illustration of different annealing effects without and with sandwiching treatment on the GeSe film. (c) XRD patterns of GeSe thin films annealed at different temperatures treated by sandwiching process. (d) Steady-state PL spectra of GeSe thin film. (e) J-V curve of the best GeSe solar cell; inset is the schematic configuration of the GeSe solar cell. (f) Histogram of the $V_{O C}$ values for GeSe solar cells. Reprinted with permission from ref. 56. Copyright 2019, Royal Society of Chemistry. 

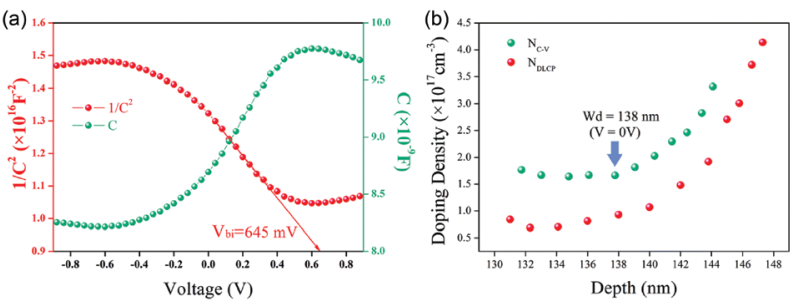

Fig. 12 (a) The $C-V$ (green) and $1 / C^{2}-V$ (red) curves for the GeSe solar cell tested at room temperature under dark conditions. (b) $C-V$ profiling and DLCP profiling for the GeSe solar cell. Reprinted with permission from ref. 56. Copyright 2019, Royal Society of Chemistry.

This result was much larger than the interfacial defect in the reported $\mathrm{ZnO} / \mathrm{Sb}_{2} \mathrm{Se}_{3}$ device $\left(3.77 \times 10^{11} \mathrm{~cm}^{-2}\right),{ }^{23}$ indicating that the interfacial defects should be mainly responsible for the low device performance.

These results display that GeSe thin-film solar cells have attracted more and more attention since the first report by our group. The best efficiency is $1.48 \%$ while the highest $V_{\mathrm{OC}}$ is higher than $450 \mathrm{mV}$, a surprising value for an emerging narrowgap material. The high $V_{\text {OC }}$ indicates that there is great room for improvement of GeSe thin-film solar cells.

\section{Conclusion and outlook}

In this paper, we present a brief review about the fundamental properties of GeSe, preparation of GeSe thin films and the relevant progress in GeSe-based thin-film solar cells. GeSe is a non-toxic, earth-abundant, and binary component. As a photovoltaic absorber material, GeSe possesses high absorption coefficient, suitable bandgap, high carrier mobility and relatively long carrier lifetime. These features make GeSe promising for thinfilm solar cells. There have been many reports about its photovoltaic application from theory to experiment. The highest efficiency of GeSe solar cells is $1.48 \%$ with a superstrate structure of ITO/CdS/GeSe/Au, where the GeSe thin films are fabricated by the RTS method. Although this preliminary efficiency is low, the result is still very encouraging considering that the first GeSe solar cell has just been reported since 2017 and there is only very limited optimization work done.

Considering the high theoretical efficiency limit of $\sim 30 \%$ for GeSe solar cells, there is still much room to further improve the efficiency of GeSe thin-film solar cells when compared to the mainstream thin-film photovoltaics such as CdTe and CIGS. The following strategies may give guidance on optimizing the GeSe thin-film solar cells to their full performance potential:

(1) Exploring new film preparation methods to further improve the GeSe film quality. For example, Tang et al. reported that vapor transport deposition (VTD) slowed the deposition of $\mathrm{Sb}_{2} \mathrm{Se}_{3}$ film, hence enabling less film defects and superior to the high deposition rate of the RTS method. ${ }^{24}$ Such methods can be attempted to slow the growth of GeSe on the substrate.

(2) Orientation control of GeSe thin films. GeSe shows obvious anisotropy due to its layered crystal structure. To realize better carrier transport, the crystal growth should be perpendicular to the substrate, providing efficient transport through covalent bonds instead of the poor hopping between the layers. ${ }^{22,67}$ Many strategies can be used to control the orientation, such as optimizing the substrate temperature and modifying the surface of the substrate.

(3) Looking for the best-matched buffer layer. The ideal buffer layer should satisfy two conditions: (i) the buffer layer forms perfect band alignment with GeSe to increase $V_{\text {OC }}$ and carrier extraction; (ii) the lattice constant and thermal expansion coefficient of the buffer layer are consistent with that of GeSe to decrease interfacial defects. ${ }^{95,96}$

(4) Introducing hole transport materials (HTM). A suitable HTM not only boosts the carrier collection efficiency, but also minimizes carrier recombination loss at the back contact. ${ }^{97-100}$ There are lots of HTMs used in perovskite solar cells, which may be available in GeSe thin-film solar cells.

(5) Constructing the substrate structure of GeSe solar cells. All the reported GeSe solar cells adopt superstrate configuration due to its simple structure, limiting the choice of substrates to transparent materials. The inverted structure of the substrate configuration allows the use of flexible plastic or metal foils, fully utilizing the merit of thin films for flexible solar cells. ${ }^{101,102}$

As an innovative absorber material, GeSe has only been investigated for three years, and there is still considerable work to do. While the above various challenges and opportunities are addressed, we believe that the efficiency of GeSe thin-film solar cells can be enhanced dramatically. More and more attention will be paid to this promising GeSe thin-film solar cell.

\section{Conflicts of interest}

There are no conflicts to declare.

\section{Acknowledgements}

This work is supported by the National Natural Science Foundation of China (Grants 21922512, 21875264), and the Youth Innovation Promotion Association CAS (2017050).

\section{Notes and references}

1 R. B. Bergmann, C. Berge, T. J. Rinke, J. Schmidt and J. H. Werner, Advances in monocrystalline Si thin film solar cells by layer transfer, Sol. Energy Mater. Sol. Cells, 2002, 74, 213.

2 J. H. Petermann, D. Zielke, J. Schmidt, F. Haase, E. G. Rojas and R. Brendel, 19\%-efficient and $43 \mu \mathrm{m}$-thick crystalline Si solar cell from layer transfer using porous silicon, Prog. Photovoltaics, 2012, 20, 1.

3 A. Goodrich, P. Hacke, Q. Wang, B. Sopori, R. Margolis, T. L. James and M. Woodhouse, A wafer-based monocrystalline silicon photovoltaics road map: utilizing known technology improvement opportunities for further reductions in manufacturing costs, Sol. Energy Mater. Sol. Cells, 2013, 114, 110. 
4 M. Taguchi, K. Kawamoto, S. Tsuge, T. Baba, H. Sakata, M. Morizane, K. Uchihashi, N. Nakamura, S. Kiyama and O. Oota, HITTM cells-high-efficiency crystalline Si cells with novel structure, Prog. Photovoltaics, 2000, 8, 503.

5 S. Philipps, F. Ise and W. Warmuth, Fraunhofer ISE: photovoltaics report, https://www.ise.fraunhofer.de/content/ dam/ise/de/documents/publications/studies/PhotovoltaicsReport.pdf.

6 K. Yoshikawa, W. Yoshida, T. Irie, H. Kawasaki, K. Konishi, H. Ishibashi, T. Asatani, D. Adachi, M. Kanematsu, H. Uzu and K. Yamamoto, Exceeding conversion efficiency of $26 \%$ by heterojunction interdigitated back contact solar cell with thin film Si technology, Sol. Energy Mater. Sol. Cells, 2017, 173, 37.

7 A. Shah, P. Torres, R. Tscharner, N. Wyrsch and H. Keppner, Photovoltaic technology: the case for thin-film solar cells, Science, 1999, 285, 692.

8 T. J. Huang, X. Yin, G. Qi and H. Gong, CZTS-based materials and interfaces and their effects on the performance of thin film solar cells, Phys. Status Solidi RRL, 2014, 08, 735.

9 NREL, Best research-cell efficiency chart, https://www.nrel. gov/pv/cell-efficiency.html, (accessed 20191106).

10 Y. Hirai, Y. Kurokawa and A. Yamada, Numerical study of $\mathrm{Cu}(\mathrm{In}, \mathrm{Ga}) \mathrm{Se}_{2}$ solar cell performance toward $23 \%$ conversion efficiency, Jpn. J. Appl. Phys., 2013, 53, 012301.

11 A. Chirilă, S. Buecheler, F. Pianezzi, P. Bloesch, C. Gretener, A. R. Uhl, C. Fella, L. Kranz, J. Perrenoud, S. Seyrling, R. Verma, S. Nishiwaki, Y. E. Romanyuk, G. Bilger and A. N. Tiwari, Highly efficient $\mathrm{Cu}(\mathrm{In}, \mathrm{Ga}) \mathrm{Se}_{2}$ solar cells grown on flexible polymer films, Nat. Mater., 2011, 10, 857.

12 K. Zeng, D.-J. Xue and J. Tang, Antimony selenide thin-film solar cells, Semicond. Sci. Technol., 2016, 31, 063001.

13 H. S. Kim, J. Y. Seo and N. G. Park, Material and device stability in perovskite solar cells, ChemSusChem, 2016, 9, 2528.

14 N. Aristidou, C. Eames, I. Sanchez-Molina, X. Bu, J. Kosco, M. S. Islam and S. A. Haque, Fast oxygen diffusion and iodide defects mediate oxygen-induced degradation of perovskite solar cells, Nat. Commun., 2017, 8, 15218.

15 S.-C. Liu, Z. Li, Y. Yang, X. Wang, Y.-X. Chen, D.-J. Xue and J.-S. $\mathrm{Hu}$, Investigation of oxygen passivation for highperformance all-inorganic perovskite solar cells, J. Am. Chem. Soc., 2019, 141, 18075.

16 P. Sinsermsuksakul, L. Sun, S. W. Lee, H. H. Park, S. B. Kim, C. Yang and R. G. Gordon, Overcoming efficiency limitations of SnS-based solar cells, Adv. Energy Mater., 2014, 4, 1400496.

17 V. Steinmann, R. Jaramillo, K. Hartman, R. Chakraborty, R. E. Brandt, J. R. Poindexter, Y. S. Lee, L. Sun, A. Polizzotti, H. H. Park, R. G. Gordon and T. Buonassisi, 3.88\% efficient tin sulfide solar cells using congruent thermal evaporation, Adv. Mater., 2014, 26, 7488.

18 H. H. Park, R. Heasley, L. Sun, V. Steinmann, R. Jaramillo, K. Hartman, R. Chakraborty, P. Sinsermsuksakul, D. Chua, T. Buonassisi and R. G. Gordon, Co-optimization of SnS absorber and $\mathrm{Zn}(\mathrm{O}, \mathrm{S})$ buffer materials for improved solar cells, Prog. Photovoltaics, 2015, 23, 901.
19 V. R. Minnam Reddy, G. Lindwall, B. Pejjai, S. Gedi, T. R. R. Kotte, M. Sugiyama, Z.-K. Liu and C. Park, $\alpha$-SnSe thin film solar cells produced by selenization of magnetron sputtered tin precursors, Sol. Energy Mater. Sol. Cells, 2018, 176, 251.

20 H.-S. Yun, B.-w. Park, Y. C. Choi, J. Im, T. J. Shin and S. I. Seok, Efficient nanostructured $\mathrm{TiO}_{2} / \mathrm{SnS}$ heterojunction solar cells, Adv. Energy Mater., 2019, 9, 1901343.

21 Y. Zhou, M. Leng, Z. Xia, J. Zhong, H. Song, X. Liu, B. Yang, J. Zhang, J. Chen, K. Zhou, J. Han, Y. Cheng and J. Tang, Solution-processed antimony selenide heterojunction solar cells, Adv. Energy Mater., 2014, 4, 1301846.

22 Y. Zhou, L. Wang, S. Chen, S. Qin, X. Liu, J. Chen, D.-J. Xue, M. Luo, Y. Cao, Y. Cheng, E. H. Sargent and J. Tang, Thin-film $\mathrm{Sb}_{2} \mathrm{Se}_{3}$ photovoltaics with oriented one-dimensional ribbons and benign grain boundaries, Nat. Photonics, 2015, 9, 409.

23 L. Wang, D.-B. Li, K. Li, C. Chen, H.-X. Deng, L. Gao, Y. Zhao, F. Jiang, L. Li, F. Huang, Y. He, H. Song, G. Niu and J. Tang, Stable 6\%-efficient $\mathrm{Sb}_{2} \mathrm{Se}_{3}$ solar cells with a ZnO buffer layer, Nat. Energy, 2017, 2, 17046.

24 X. Wen, C. Chen, S. Lu, K. Li, R. Kondrotas, Y. Zhao, W. Chen, L. Gao, C. Wang, J. Zhang, G. Niu and J. Tang, Vapor transport deposition of antimony selenide thin film solar cells with 7.6\% efficiency, Nat. Commun., 2018, 9, 2179.

25 Z. Li, X. Liang, G. Li, H. Liu, H. Zhang, J. Guo, J. Chen, K. Shen, X. San, W. Yu, R. E. I. Schropp and Y. Mai, 9.2\%efficient core-shell structured antimony selenide nanorod array solar cells, Nat. Commun., 2019, 10, 125.

26 E. Zimmermann, T. Pfadler, J. Kalb, J. A. Dorman, D. Sommer, G. Hahn, J. Weickert and L. Schmidt-Mende, Toward high-efficiency solution-processed planar heterojunction $\mathrm{Sb}_{2} \mathrm{~S}_{3}$ solar cells, Adv. Sci., 2015, 2, 1500059.

27 R. Kondrotas, C. Chen and J. Tang, $\mathrm{Sb}_{2} \mathrm{~S}_{3}$ solar cells, Joule, 2018, 2, 857.

28 D.-J. Xue, B. Yang, Z.-K. Yuan, G. Wang, X. Liu, Y. Zhou, L. Hu, D. Pan, S. Chen and J. Tang, $\mathrm{CuSbSe}_{2}$ as a potential photovoltaic absorber material: studies from theory to experiment, Adv. Energy Mater., 2015, 5, 1501203.

29 A. S. Kshirsagar and P. K. Khanna, $\mathrm{CuSbSe}{ }_{2} / \mathrm{TiO}_{2}$ : novel type-II heterojunction nano-photocatalyst, Mater. Chem. Front., 2019, 3, 437.

$30 \mathrm{Y}$. Hameş and S. Eren $\mathrm{San}, \mathrm{CdO} / \mathrm{Cu}_{2} \mathrm{O}$ solar cells by chemical deposition, Sol. Energy, 2004, 77, 291.

31 K. P. Musselman, A. Marin, L. Schmidt-Mende and J. L. MacManus-Driscoll, Incompatible length scales in nanostructured $\mathrm{Cu}_{2} \mathrm{O}$ solar cells, Adv. Funct. Mater., 2012, 22, 2202.

$32 \mathrm{Z}$. Zang, Efficiency enhancement of $\mathrm{ZnO} / \mathrm{Cu}_{2} \mathrm{O}$ solar cells with well oriented and micrometer grain sized $\mathrm{Cu}_{2} \mathrm{O}$ films, Appl. Phys. Lett., 2018, 112, 042106.

33 D.-J. Xue, S.-C. Liu, C.-M. Dai, S. Chen, C. He, L. Zhao, J.-S. Hu and L.-J. Wan, GeSe thin-film solar cells fabricated by self-regulated rapid thermal sublimation, J. Am. Chem. Soc., 2017, 139, 958.

34 W. Shockley and H. J. Queisser, Detailed balance limit of Efficiency of p-n Junction Solar Cells, J. Appl. Phys., 1961, 32, 510 . 
35 L. C. Gomes and A. Carvalho, Phosphorene analogues: isoelectronic two-dimensional group-IV monochalcogenides with orthorhombic structure, Phys. Rev. B: Condens. Matter Mater. Phys., 2015, 92, 085406.

36 Z. Ma, B. Wang, L. Ou, Y. Zhang, X. Zhang and Z. Zhou, Structure and properties of phosphorene-like IV-VI 2D materials, Nanotechnology, 2016, 27, 415203.

37 Z. Li, X. Wang, W. Shi, X. Xing, D.-J. Xue and J.-S. Hu, Strain-engineering the electronic properties and anisotropy of $\mathrm{GeSe}_{2}$ monolayers, RSC Adv., 2018, 8, 33445.

38 Y. Yang, S.-C. Liu, X. Wang, Z. Li, Y. Zhang, G. Zhang, D.-J. Xue and J.-S. Hu, Polarization-sensitive ultraviolet photodetection of anisotropic $2 \mathrm{D} \mathrm{GeS}_{2}$, Adv. Funct. Mater., 2019, 29, 1900411.

39 X. Wang, Y. Li, L. Huang, X.-W. Jiang, L. Jiang, H. Dong, Z. Wei, J. Li and W. Hu, Short-wave near-infrared linear dichroism of two-dimensional germanium selenide, J. Am. Chem. Soc., 2017, 139, 14976.

40 H. Zhao, Y. Mao, X. Mao, X. Shi, C. Xu, C. Wang, S. Zhang and D. Zhou, Band structure and photoelectric characterization of GeSe monolayers, Adv. Funct. Mater., 2018, 28, 1704855.

41 Z. Yang, L. Liao, F. Gong, F. Wang, Z. Wang, X. Liu, X. Xiao, W. $\mathrm{Hu}, \mathrm{J}$. He and X. Duan, $\mathrm{WSe}_{2} / \mathrm{GeSe}$ heterojunction photodiode with giant gate tunability, Nano Energy, 2018, 49, 103.

42 X. Zhou, X. Hu, B. Jin, J. Yu, K. Liu, H. Li and T. Zhai, Highly anisotropic GeSe nanosheets for phototransistors with ultrahigh photoresponsivity, Adv. Sci., 2018, 5, 1800478.

43 D.-J. Xue, J. Tan, J.-S. Hu, W. Hu, Y.-G. Guo and L.-J. Wan, Anisotropic photoresponse properties of single micrometersized GeSe nanosheet, Adv. Mater., 2012, 24, 4528.

44 Y. Yang, S.-C. Liu, W. Yang, Z. Li, Y. Wang, X. Wang, S. Zhang, Y. Zhang, M. Long, G. Zhang, D.-J. Xue, J.-S. Hu and L.-J. Wan, Air-stable in-plane anisotropic $\mathrm{GeSe}_{2}$ for highly polarization-sensitive photodetection in short wave region, J. Am. Chem. Soc., 2018, 140, 4150.

45 Y. Yang, X. Wang, S.-C. Liu, Z. Li, Z. Sun, C. Hu, D.-J. Xue, G. Zhang and J.-S. Hu, Weak interlayer interaction in 2D anisotropic $\mathrm{GeSe}_{2}, A d v$. Sci., 2019, 6, 1801810.

46 J. Liu, Y. Zhou, Y. Lin, M. Li, H. Cai, Y. Liang, M. Liu, Z. Huang, F. Lai, F. Huang and W. Zheng, Anisotropic photoresponse of the ultrathin GeSe nanoplates grown by rapid physical vapor deposition, ACS Appl. Mater. Interfaces, 2019, 11, 4123.

47 H.-W. Ahn, D. S. Jeong, B.-K. Cheong, S.-D. Kim, S.-Y. Shin, H. Lim, D. Kim and S. Lee, A study on the scalability of a selector device using threshold switching in $\mathrm{Pt} / \mathrm{GeSe} / \mathrm{Pt}$, ECS Solid State Lett., 2013, 2, N31.

48 S.-Y. Shin, J. M. Choi, J. Seo, H.-W. Ahn, Y. G. Choi, B.-K. Cheong and S. Lee, The effect of doping $\mathrm{Sb}$ on the electronic structure and the device characteristics of ovonic threshold switches based on Ge-Se, Sci. Rep., 2014, 4, 7099.

49 W. Kim, C. Yoo, E.-S. Park, M. Ha, J. W. Jeon, G. S. Kim, K. S. Woo, Y. K. Lee and C. S. Hwang, Electroforming-free bipolar resistive switching in GeSe thin films with a Ticontaining electrode, ACS Appl. Mater. Interfaces, 2019, 11, 38910.

50 B. Song, H. Xu, S. Liu, H. Liu, Q. Liu and Q. Li, An ovonic threshold switching selector based on Se-rich GeSe chalcogenide, Appl. Phys. A: Mater. Sci. Process., 2019, 125, 772.

51 K. Wang, D. Huang, L. Yu, K. Feng, L. Li, T. Harada, S. Ikeda and F. Jiang, Promising GeSe nanosheet-based thin-film photocathode for efficient and stable overall solar water splitting, ACS Catal., 2019, 9, 3090.

52 L. Liu, Q. Yang, Z. Wang, H. Ye, X. Chen, X. Fan and G. Zhang, High selective gas detection for small molecules based on germanium selenide monolayer, Appl. Surf. Sci., 2018, 433, 575.

53 S. M. Yoon, H. J. Song and H. C. Choi, p-type semiconducting GeSe combs by a vaporization-condensation-recrystallization (VCR) process, Adv. Mater., 2010, 22, 2164.

54 M. Brahma, A. Kabiraj, D. Saha and S. Mahapatra, Scalability assessment of group-IV mono-chalcogenide based tunnel FET, Sci. Rep., 2018, 8, 5993.

55 W. C. Yap, Z. Yang, M. Mehboudi, J.-A. Yan, S. BarrazaLopez and W. Zhu, Layered material GeSe and vertical GeSe/MoS 2 p-n heterojunctions, Nano Res., 2018, 11, 420.

56 B. Chen, G. Chen, W. Wang, H. Cai, L. Yao, S. Chen and Z. Huang, Magnetron sputtering deposition of GeSe thin films for solar cells, Sol. Energy, 2018, 176, 98.

57 B. Chen, Y. Ruan, J. Li, W. Wang, X. Liu, H. Cai, L. Yao, J.-M. Zhang, S. Chen and G. Chen, Highly oriented GeSe thin film: self-assembly growth via the sandwiching post-annealing treatment and its solar cell performance, Nanoscale, 2019, 11, 3968.

58 P. D. Antunez, J. J. Buckley and R. L. Brutchey, Tin and germanium monochalcogenide IV-VI semiconductor nanocrystals for use in solar cells, Nanoscale, 2011, 3, 2399.

$59 \mathrm{H}$. Wiedemeier and P. A. Siemers, The thermal expansion and high temperature transformation of GeSe, Z. Anorg. Allg. Chem., 1975, 411, 90.

60 M. D. Coutts and E. R. Levin, Phase transformation of $\mathrm{As}_{2} \mathrm{Se}_{3}$ and $\mathrm{Sb}_{2} \mathrm{Se}_{3}$ films, J. Appl. Phys., 1967, 38, 4039.

61 R. Balasubramanian and W. R. Wilcox, Mechanical properties of CdTe, Mater. Sci. Eng., B, 1993, 16, 1.

62 H. Ipser, M. Gambino and W. Schuster, The germaniumselenium phase diagram, Monatsh. Chem., 1982, 113, 389.

63 Y. Yang, S.-C. Liu, Y. Wang, M. Long, C.-M. Dai, S. Chen, B. Zhang, Z. Sun, Z. Sun, C. Hu, S. Zhang, L. Tong, G. Zhang, D.-J. Xue and J.-S. Hu, In-plane optical anisotropy of low-symmetry 2D GeSe, Adv. Opt. Mater., 2019, 7, 1801311.

64 Z. Li, X. Wang, S.-C. Liu, W. Shi, X. Xing, D.-J. Xue and J.-S. Hu, Strain-engineering the anisotropic electrical properties of low-symmetry bilayer GeSe, J. Appl. Phys., 2019, 125, 082524.

65 C. Xia, J. Du, W. Xiong, Y. Jia, Z. Wei and J. Li, A type-II GeSe/SnS heterobilayer with a suitable direct gap, superior optical absorption and broad spectrum for photovoltaic applications, J. Mater. Chem. A, 2017, 5, 13400. 
66 J. G. A. Speight, Lange's Handbook of Chemistry, McGraw Hill Book Co., New York, 2005.

67 K. Li, C. Chen, S. Lu, C. Wang, S. Wang, Y. Lu and J. Tang, Orientation engineering in low-dimensional crystalstructural materials via seed screening, Adv. Mater., 2019, 31, 1903914.

68 Y. Ishihara, Y. Ohno and I. Nakada, Anisotropic electrical properties of GeSe, Phys. Status Solidi B, 1984, 121, 407.

69 S.-C. Liu, Y. Mi, D.-J. Xue, Y.-X. Chen, C. He, X. Liu, J.-S. Hu and L.-J. Wan, Investigation of physical and electronic properties of GeSe for photovoltaic applications, $A d v$. Electron. Mater., 2017, 3, 1700141.

70 G. D. Scholes and G. Rumbles, Excitons in nanoscale systems, Nat. Mater., 2006, 5, 683.

71 C. Chen, D. C. Bobela, Y. Yang, S. Lu, K. Zeng, C. Ge, B. Yang, L. Gao, Y. Zhao, M. C. Beard and J. Tang, Characterization of basic physical properties of $\mathrm{Sb}_{2} \mathrm{Se}_{3}$ and its relevance for photovoltaics, Front. Optoelectron., 2017, 10, 18.

72 P. Zhao, H. Yang, J. Li, H. Jin, W. Wei, L. Yu, B. Huang and Y. Dai, Design of new photovoltaic systems based on twodimensional group-IV monochalcogenides for high performance solar cells, J. Mater. Chem. A, 2017, 5, 24145.

73 G. K. Solanki, M. P. Deshpande, M. K. Agarwal, P. D. Patel and S. N. Vaidya, Thermoelectric power factor measurements in GeSe single crystals grown using different transporting agents, J. Mater. Sci. Lett., 2003, 22, 985.

74 F. Ersan, H. Arkin and E. Aktürk, The effect of vacancies and the substitution of p-block atoms on single-layer buckled germanium selenide, RSC Adv., 2017, 7, 37815.

75 Y. Kim and I.-H. Choi, Optical and electrical properties of GeSe and SnSe single crystals, J. Korean Phys. Soc., 2018, 72, 238.

76 S. D. Stranks, G. E. Eperon, G. Grancini, C. Menelaou, M. J. P. Alcocer, T. Leijtens, L. M. Herz, A. Petrozza and H. J. Snaith, Electron-hole diffusion lengths exceeding 1 micrometer in an organometal trihalide perovskite absorber, Science, 2013, 342, 341.

77 S. Hino, T. Takahashi and Y. Harada, Thermally induced effects in amorphous $\mathrm{GeSe}_{2}$ and GeSe films studied by ultraviolet photoelectron spectroscopy, Solid State Commun., 1980, 35, 379.

78 M. S. Kamboj, G. Kaur, R. Thangaraj and D. K. Avasthi, Effect of heavy ion irradiation on the electrical and optical properties of amorphous chalcogenide thin films, J. Phys. D: Appl. Phys., 2002, 35, 477.

79 J. L. Bosse, I. Grishin, Y. G. Choi, B.-K. Cheong, S. Lee, O. V. Kolosov and B. D. Huey, Nanosecond switching in GeSe phase change memory films by atomic force microscopy, Appl. Phys. Lett., 2014, 104, 053109.

80 W. Kim, S. Yoo, C. Yoo, E.-S. Park, J. Jeon, Y. J. Kwon, K. S. Woo, H. J. Kim, Y. K. Lee and C. S. Hwang, Atomic layer deposition of GeSe films using $\mathrm{HGeCl}_{3}$ and $\left[\left(\mathrm{CH}_{3}\right)_{3} \mathrm{Si}\right]_{2} \mathrm{Se}$ with the discrete feeding method for the ovonic threshold switch, Nanotechnology, 2018, 29, 365202.

81 S.-C. Liu, Y. Yang, X. Zhang, L.-B. Huang, J.-K. Sun, B. Guan, X. Li, D.-J. Xue and J.-S. Hu, Tuning the optical absorption property of GeSe thin films by annealing treatment, Phys. Status Solidi RRL, 2018, 12, 1800370.

82 D. D. Vaughn, R. J. Patel, M. A. Hickner and R. E. Schaak, Single-crystal colloidal nanosheets of GeS and GeSe, J. Am. Chem. Soc., 2010, 132, 15170.

83 D. Vaughn, D. Sun, S. M. Levin, A. J. Biacchi, T. S. Mayer and R. E. Schaak, Colloidal synthesis and electrical properties of GeSe nanobelts, Chem. Mater., 2012, 24, 3643.

84 L. Shi, Y. Li and Y. Dai, Preparation, formation mechanism, and photoresponse properties of GeSe microtubes with a rectangular cross section, ChemPlusChem, 2015, 80, 630.

85 M. Rusek, G. Bendt, C. Wölper, D. Bläser and S. Schulz, Intramolecularly-stabilized group 14 alkoxides-promising precursors for the synthesis of group 14-chalcogenides by hot-injection method, Z. Anorg. Allg. Chem., 2017, 643, 676.

86 D. Sun and R. E. Schaak, Solution-mediated growth of two-dimensional SnSe@GeSe nanosheet heterostructures, Chem. Mater., 2017, 29, 817.

87 Z.-Q. Fan, X.-W. Jiang, Z. Wei, J.-W. Luo and S.-S. Li, Tunable electronic structures of GeSe nanosheets and nanoribbons, J. Phys. Chem. C, 2017, 121, 14373.

88 G. Shi and E. Kioupakis, Anisotropic spin transport and strong visible-light absorbance in few-layer SnSe and GeSe, Nano Lett., 2015, 15, 6926.

89 P. Mishra, H. Lohani, A. K. Kundu, R. Patel, G. K. Solanki, K. S. R. Menon and B. R. Sekhar, Electronic structure of germanium selenide investigated using ultra-violet photoelectron spectroscopy, Semicond. Sci. Technol., 2015, 30, 075001.

90 X. Lv, W. Wei, C. Mu, B. Huang and Y. Dai, Twodimensional GeSe for high performance thin-film solar cells, J. Mater. Chem. A, 2018, 6, 5032.

91 M. C. Scharber, D. Mühlbacher, M. Koppe, P. Denk, C. Waldauf, A. J. Heeger and C. J. Brabec, Design rules for donors in bulk-heterojunction solar cells-towards $10 \%$ energy-conversion efficiency, Adv. Mater., 2006, 18, 789.

92 Y. Mao, C. Xu, J. Yuan and H. Zhao, A two-dimensional GeSe/SnSe heterostructure for high performance thin-film solar cells, J. Mater. Chem. A, 2019, 7, 11265.

93 G.-J. Hou, D.-L. Wang, R. Ali, Y.-R. Zhou, Z.-G. Zhu and G. $\mathrm{Su}, \mathrm{CH}_{3} \mathrm{NH}_{3} \mathrm{PbI}_{3} / \mathrm{GeSe}$ bilayer heterojunction solar cell with high performance, Sol. Energy, 2018, 159, 142.

94 J. T. Heath, J. D. Cohen and W. N. Shafarman, Bulk and metastable defects in $\mathrm{CuIn}_{1-x} \mathrm{Ga}_{x} \mathrm{Se}_{2}$ thin films using drivelevel capacitance profiling, J. Appl. Phys., 2004, 95, 1000.

95 L. Sun, R. Haight, P. Sinsermsuksakul, S. B. Kim, H. H. Park and R. G. Gordon, Band alignment of $\mathrm{SnS} / \mathrm{Zn}(\mathrm{O}, \mathrm{S})$ heterojunctions in SnS thin film solar cells, Appl. Phys. Lett., 2013, 103, 181904.

96 Q. Jiang, X. Zhang and J. You, $\mathrm{SnO}_{2}$ : a wonderful electron transport layer for perovskite solar cells, Small, 2018, 14, 1801154.

97 M. Bernechea, N. C. Miller, G. Xercavins, D. So, A. Stavrinadis and G. Konstantatos, Solution-processed solar cells based on environmentally friendly $\mathrm{AgBiS}_{2}$ nanocrystals, Nat. Photonics, 2016, 10, 521.

98 C. Chen, L. Wang, L. Gao, D. Nam, D. Li, K. Li, Y. Zhao, C. Ge, H. Cheong, H. Liu, H. Song and J. Tang, 6.5\% 
certified efficiency $\mathrm{Sb}_{2} \mathrm{Se}_{3}$ solar cells using $\mathrm{PbS}$ colloidal quantum dot film as hole-transporting layer, ACS Energy Lett., 2017, 2, 2125.

99 Q.-Q. Ge, J.-Y. Shao, J. Ding, L.-Y. Deng, W.-K. Zhou, Y.-X. Chen, J.-Y. Ma, L.-J. Wan, J. Yao, J.-S. Hu and Y.-W. Zhong, A two-dimensional hole-transporting material for high-performance perovskite solar cells with $20 \%$ average efficiency, Angew. Chem., Int. Ed., 2018, 57, 10959.

100 K. Li, S. Wang, C. Chen, R. Kondrotas, M. Hu, S. Lu, C. Wang, W. Chen and J. Tang, 7.5\% n-i-p $\mathrm{Sb}_{2} \mathrm{Se}_{3}$ solar cells with CuSCN as a hole-transport layer, J. Mater. Chem. A, 2019, 7, 9665.

101 Z. Li, X. Chen, H. Zhu, J. Chen, Y. Guo, C. Zhang, W. Zhang, X. Niu and Y. Mai, $\mathrm{Sb}_{2} \mathrm{Se}_{3}$ thin film solar cells in substrate configuration and the back contact selenization, Sol. Energy Mater. Sol. Cells, 2017, 161, 190.

102 G. Li, Z. Li, X. Liang, C. Guo, K. Shen and Y. Mai, Improvement in $\mathrm{Sb}_{2} \mathrm{Se}_{3}$ solar cell efficiency through band alignment engineering at the buffer/absorber interface, ACS Appl. Mater. Interfaces, 2019, 11, 828. 